Relations industrielles

Industrial Relations

\title{
Kawanishi, Hirosuke, sous la direction de, The Human Face of Industrial Conflict in Post-War Japan
}

\section{Mona-Josée Gagnon}

Volume 55, numéro 1, 2000

URI : https://id.erudit.org/iderudit/051302ar

DOI : https://doi.org/10.7202/051302ar

Aller au sommaire du numéro

Éditeur(s)

Département des relations industrielles de l'Université Laval

ISSN

0034-379X (imprimé)

1703-8138 (numérique)

Découvrir la revue

Citer ce compte rendu

Gagnon, M.-J. (2000). Compte rendu de [Kawanishi, Hirosuke, sous la direction de, The Human Face of Industrial Conflict in Post-War Japan]. Relations industrielles / Industrial Relations, 55(1), 185-187.

https://doi.org/10.7202/051302ar

Tous droits réservés (C) Département des relations industrielles de l'Université Laval, 2000
Ce document est protégé par la loi sur le droit d'auteur. L'utilisation des services d'Érudit (y compris la reproduction) est assujettie à sa politique d'utilisation que vous pouvez consulter en ligne.

https://apropos.erudit.org/fr/usagers/politique-dutilisation/ 


\section{The Human Face of Industrial Conflict in Post-War Japan}

sous la direction de Hirosuke KAWANISHI, London et New York : Kegan

Paul International, 1999, 287 p., ISBN 0-7103-0563-X.

Les analyses comparatistes des systèmes de relations du travail ne nous permettent pas toujours de connaître l'origine sociohistorique des processus et structures étudiés. Pour plusieurs, le syndicalisme japonais rime avec emploi à vie (ce qui est de moins en moins vrai) et localisme (ce qui est toujours vrai). Mais pourquoi ? Comment? Peu de sources sont accessibles au lecteur qui ne lit pas le japonais (ce qui est présumément le cas de $99 \%$ des lecteurs et lectrices de cette revue, incluant la soussignée).

L'auteur de l'ouvrage enseigne la sociologie à l'Université de Waseda. Il a publié en 1992 chez le même éditeur Enterprise Unionism in Japan. Son approche se démarque des quelques articles et ouvrages qui nous sont accessibles sur le syndicalisme japonais.

L'ouvrage à l'étude s'inscrit dans une perspective critique, selon laquelle patronat et salariés sont séparés par des intérêts contradictoires, les conflits de travail apparaissant comme autant de résolutions non institutionnalisées de cette contradiction et les systèmes de relations du travail ayant pour rôle essentiel de gérer ces contradictions. De plus, l'auteur condamne les analyses culturalistes (que n'a-t-on entendu parler du confucianisme et du féodalisme tardif s'agissant du Japon ?). Il privilégie les approches fondées sur la sociohistoricité. Cet ouvrage constitue donc une introduction à la construction sociopolitique du système de relations du travail japonais et, partant, du syndicalisme nippon, construction qui pour l'essentiel s'est effectuée dans l'immédiat après-guerre.

On peut lire le premier ouvrage (1992) traduit en anglais de l'auteur pour comprendre le fonctionnement contemporain $\mathrm{du}$ syndicalisme japonais. Un article, paru dans Sociologie et Sociétés (collaboration R. Mouer, vol. XXX, $\mathrm{n}^{\circ} 2,1998,95-112$ ), propose un bilan récent du syndicalisme japonais. L'ouvrage à l'étude, et c'est ce qui fait son intérêt, nous ramène au moment et aux lieux où se construisit l'actuel système de relations du travail. Et qui l'a lu ne pourra plus jamais entretenir une vision caricaturale du syndicalisme japonais. C'est l'histoire d'une construction sociopolitique, c'est aussi 1'histoire d'une répression à très grande échelle, répression dont le principal orchestrateur fut le GHQ (General Headquarters of the Allied Forces), dirigé par le général MacArthur. En voilà assez pour nous sentir concernés.

Kawanishi, dans le cadre de son enseignement, organisa en 1983 une série de séminaires au cours desquels des vétérans (septuagénaires) des luttes syndicales de l'après-guerre livraient une conférence-témoignage en parallèle avec une mise en situation proposée par un universitaire. Ces témoignages furent enregistrés et retranscrits. L'intention était d'informer les étudiants et les étudiantes de construire des matériaux historiques et d'ouvrir l'université à la société civile (avant-propos). Les choix furent difficiles, les survivants étant clairsemés et l'intention étant de fournir une image représentative des différentes tendances existant à l'époque au sein du syndicalisme japonais. De ce point de vue, c'est réussi. Un familier du syndicalisme retrouve aisément les grands clivages qui ont marqué l'histoire de ce dernier. L'ouvrage a été réédité plusieurs fois au Japon (première édition 1986). La parution en langue anglaise est postérieure à celle d'une traduction en langue allemande. L'ouvrage luimême est constitué d'un avant-propos ainsi que d'un chapitre de mise en situation. Suivent sept chapitres monographiques relatifs à des conflits particuliers ; Kawanishi fait toutefois précéder chacun des témoignages d'une mise en situation 
spécifique et d'un résumé. En fin d'ouvrage, une chronologie et un lexique des noms japonais des organisations en cause fournissent au lecteur des repères utiles.

Le lecteur occidental trouvera plus d'intérêt à certains des témoignages qu'aux synthèses de l'auteur principal. Le lecteur «paresseux » en fera cependant son miel, parfaites pour la préparation d'un cours, mais manquera le meilleur, soit des témoignages incarnés, contextualisés, dont plusieurs se lisent comme des romans.

Certes, les témoignages livrés $a$ posteriori (très a posteriori, trente ou quarante ans après les faits) sont susceptibles de souffrir de bien des distorsions. La mémoire est-elle fidèle ? Les chiffres invoqués sont-ils véridiques? Je tends à penser que le directeur de publication a vérifié tous les détails de près ; ainsi les témoignages font-ils état d'une rigueur qui éveille les soupçons. Il est difficile de croire que chaque témoin a spontanément fourni un texte impeccablement catégorisé et numéroté ; par exemple : trois facteurs $1-2-3$; quatre conséquences 1-2-3-4; cinq épisodes 1 2-3-4-5. À coup sûr, l'éditeur est passé par là, a systématisé, vérifié les chiffres, mais n'a pas osé gommer quelques considérations moralisatrices sans intérêt historique. Les sept témoins, tous acteurs centraux d'événements qui ont contribué à construire la société japonaise, évoquent les styles variés des grandes figures du syndicalisme d'ici ; il y a ceux que la modestie rend inexistants et dont on ne sait qu'ils ont été sur la première ligne que parce qu'on l'a lu en introduction; il y a ces autres qui existent, certes, mais pas seuls, et qui même expriment leurs angoisses ct incertitudes passées et présentes ; enfin, il y a les inévitables de ce troisième camp qui écrivent à la première personne et qui ont mené des conflits à la façon dont on dit couramment que Christophe Colomb a découvert l'Amérique, seul devant Dieu et la postérité. Nostalgie, triomphalisme, règlements de compte sont au rendez-vous. Cette mémoire parfois acérée soulève des questions : comment peut-on reproduire, quarante ans plus tard, les discours qu'on a improvisés dans l'affolement total face à trois mille ouvriers agressifs, alors que les Japonais n'avaient pas encore inventé l'enregistreuse ? Certes, il y a une part de fabrication dans cet ouvrage, et pourtant le tout s'impose par son authenticité (diversité des points de vue notamment, mais aussi absence de censure relative à certains épanchements...). Le lecteur qui aime se prêter à une lecture au second degré y trouvera son compte.

Finalement, qu'y a-t-il à tirer de ce livre? À part l'émotion, les éléments suivants :

- Après la défaite, dans un pays exsangue dont la population avait été très éprouvée, les travailleurs (démobilisés et autres) ont été à l'origine de plusieurs conflits. Ils ont, en 19456 , remis au monde un syndicalisme que le Japon militarisé avait interdit dès 1940 ; des groupes ont même lutté pour la conservation de leurs postes par des femmes et des jeunes.

- Les puissances occupantes (symbolisées par le général MacArthur) se sont au départ proclamées pour la « démocratisation » du Japon, considéré « retardataire »; les femmes ont obtenu ainsi le droit de vote ; des lois du travail inspirées du New Deal ont été promulguées.

- Les puissances occupantes ont par la suite raidi le ton, en alliance avec le patronat et le gouvernement japonais ; la lutte au communisme se mit en branle, le Japon ayant dès 1947 reçu le douteux honneur d'être proclamé par les porte-parole américains de rempart contre la propagation du communisme en Asie; des milliers de militants politiques et syndicaux perdirent leur travail, ou dans plusieurs cas y renoncèrent, "pour sauver l'emploi des autres ». 
- La répression antisyndicale a pris la forme de congédiements, mais aussi d'interventions musclées des occupants américains, de union-busting, notamment par l'aide à la mise sur pied d'autres syndicats (second union), qui s'épanouissaient d'autant facilement que tous les leaders des premiers avaient été mis hors de combat.

- Les tentatives des travailleurs japonais de former des organisations et d'articuler des luttes sectorielles ont été mises en déroute, d'où l'avènement du company unionism, calqué sur le modèle américain ; la mise sur pied de comités d'entreprises (strictement consultatifs) ont permis à certains de diagnostiquer une certaine influence du modèle allemand.

En dernier ressort, il pourra être intéressant de consulter ce livre pour y constater que, loin d'être « en retard», les militants japonais d'après-guerre ont mis de l'avant des pratiques et revendications reprises par la suite en Occident dans les années 1970 sous le nom d'autogestion. Elles furent bien sûr réprimées par l'occupant américain, de même que fut interdite une grève générale (1 ${ }^{\text {er }}$ février 1947), cette dernière appelée dans un climat véritablement prérévolutionnaire (il était question de renverser le gouvernement). Parallèlement aux conflits dits « du travail » prirent place des manifestations populaires contre la faim ; la population était affamée, rationnée, les denrées bloquées dans des entrepôts, la récolte de riz désastreuse... C'est cette conjoncture qui donne son titre, qui peut autrement avoir l'air saugrenu, au livre: The HUMAN Face of Industrial Conflict in Post-War Japan, les conflits de travail étant aussi centrés sur l'accès à un minimum vital.

Traverse ce livre l'inéluctabilité de l'existence de diverses factions politiques au sein du mouvement syndical, habilement exacerbée par le patronat. De même que l'acharnement des forces combinées de la puissance occupante, du patronat et du gouvernement japonais pour empêcher la construction/consolidation d'un syndicalisme japonais combatif. Somme toute, ce livre est bienvenu comme antidote à un certain nombre de clichés à combattre et pour mettre de l'avant une ou deux idées-forces. Ainsi que le syndicalisme, qu'il soit d'ici ou d'ailleurs, est un produit sociohistorique, et que le syndicalisme japonais a derrière lui une histoire de militantisme $e t$ de répression qui le distinguent.

MONA-JosÉE GAGNON Université de Montréal

\section{Labor and the State in Egypt}

by Marsha Pripstein POSUSNEY, New York : Columbia University Press, 1997, 327 p., ISBN 02-3110-692-0.

Structural adjustment programs (SAP) in African countries have been a historical turning point. For labour and its organizations, SAPs have been a test of their capacity to respond to radical environmental change after the long period of relative stability following independence. For students in industrial relations and labour studies, SAPs are an opportunity for comparative analysis of labour's response to these changes. They also raise questions about the old para- digms concerning the relationship between the state, labour, employers and the general society on issues of economic development and nation building.

Most analyses concern only the impact of SAPs on workers, their unions and their working conditions (B. Tidjani, "African Unions under Structural Adjustment Programs," Relations industrielles/ Industrial Relations, Vol. 53, No. 2, 1998). Posusney's Labor and the State in Egypt offers both an understanding of 